\title{
Procedural Similarity and Its Effect on Transfer
}

\author{
Huda Saifaddin \\ Department of Psychology King Abdul Aziz University, Jeddah, Saudi Arabia
}

\begin{abstract}
Analogical problem solving involves transferring information from a particular domain (i.e., the analogue or source) to another (i.e., the target). The success of any transfer process depends on the modality of representation (verbal or pictorial) and the level of abstraction or similarity shared between the source and target problems.

The Experiment predicted that procedural similarity will influence transfer more than the principle and strategy levels. One hundred and fifty-six female undergraduate Saudi students were randomly assigned to five experimental and one control condition that differed in the extent to which the pictorially depicted source shared a concrete procedure with the target. Contrary to prediction no significant difference in transfer performance among the different levels of similarity was found. Retrospective reports of participants indicated that the pictures lacked clarity and tended to process the information in the pictures in the $\mathrm{R}$ to $\mathrm{L}$ instead of $\mathrm{L}$ to $\mathrm{R}$ sequence which affected the interpretation of the process depicted.
\end{abstract} similarity

Index Terms - Analogy, Problem Solving, Transfer, procedural

\section{Introduction}

Problem solving by Analogy is a mental process of transferring information from a particular domain (i.e., the source) to another (i.e., the target). The success of this transfer process is contingent on the ease with which acquired information from the source is retrieved and applied to solve similar problems. Failures in the transfer process often occur when individuals are unable to apply the solution they previously learned.

Analogy is considered an effective cognitive tool for learning and conceptual change, The history of work on analogical problem solving is drafted with focus on various inputs from cognitive science (Gentner 1983) cognitive psychology (Chen \& Mo 2004), artificial intelligence (Daives, Goel \& Nersassian 2003) educational psychology (Van meter et al 2006) which reflects on its importance. The investigation of the mechanisms of analogical problem solving has yielded a great deal of progress over the past twenty years.

Theories of analogical problem solving (for example, Gentner 1983 and Holyoak \& Thagard 1995) held that analogy is characterized by the mapping of relations between the objects rather than attributes of objects from base to target problem Kurtz, Boukrina \& Gentner (2013). They used complex tasks that provided a scope for examining how the relational structures between the base and target problems affect the mapping and transfer processes (Gick \& Holyoak 1980; 1983).

A central feature of both Gentner's and Holyoak's theory concerns the representational structure of the problem. A common feature shared by Gentner's and Holyoak's theories is that the effectiveness of the mapping process between the base and target domains is largely determined by the way in which a problem is represented at the structural level. For example, Holyoak considered level of abstraction or similarity, shared between the source and target, important in analogical transfer, whereas Gentner \& Markman (1993, 1994) refer to the similarity of correspondences between structured representations as affecting the mapping process.

Analogical problem solving often involves reconceptualizing a problem or a strategy for its solution in a totally new way that emerges by detecting and combining relevant old and new information. The probability of successfully solving a problem by analogy is greatly determined by the representation of the source and target problems, that is, the information or concepts involved (e.g. weighing or measuring), the organization of this information and the clarity of goals and constraints. It also depends on the degree of diversity (or similarity or level of abstraction) shared between the source and the target problems. Isomorphic problems usually involved the process of identifying the underlying structural isomorphism of problems and applying the idea or the method to solve another problem (e.g. from a textbook to a problem on a test).

Zhang and Norman (1994) consider external representations as memory aids that anchor and structure cognitive behavior that helps determine what information to select and how to be implemented. Pictorial forms of representation were very often used in educational contexts to enhance learning outcomes. Ainsworth and Loizou (2003) reported that participants given information about the human circulatory system in diagrams learnt more than those given texts. Students given diagrams also performed better than students given texts, particularly on more difficult knowledge inference questions. The study revealed that the process of learning is enhanced by graphical representation that helped in understanding and manipulating information. They used this evidence to claim that diagrams differentially aid learning. On the other hand, relatively few studies have used pictorial representations in analogical reasoning as source analogues. In their famous study, Gick and Holyoak $(1980 ; 1983)$ found that only $10 \%$ of the participants generated a solution when no source analogue was given, and about $30 \%$ gave the correct solution to a highly dissimilar source problem. Finally, when the source was given in diagrammatic form the post-hint performance was $70 \%$. Pedone, et al. (2001) thus concluded that diagrams cannot be reliably accessed as source analogues without hints on the basis that diagrams are not encoded in terms of concepts that could link them to a verbal target problem.

Chen (2002) took the research a step further by representing the source problem at different levels of abstraction or similarity with the target problem. The study examined the effect of diagrammatical representation 
depicting a process in procedural similarity, as compared to the strategy and principle levels of similarity between the source and target, on transfer. Chen's study differed from that of Holyoak and Pedone et al. in two ways: firstly, he schematically represented a problem involving a process at three different levels of similarity with the target problem, and secondly, the source problem (weighing large objects) required the participants to figure out and discover the underlying principle and solve it. Although, Chen found, as expected, that the procedural level of similarity was more effective, he also discovered that the major barrier to successful solution in analogical problem solving, involving a process, was failure in the executing process. That is, failure to apply the solution discovered in the source problem to the target problem.

Psychologists and cognitive scientists have always been interested in questions such as why people often fail to solve problems although they possess all the necessary information, which could either be part of their past experience (retrieved from memory) or be provided by the environment during the problem solving process. In both cases people can differ significantly in the degree to which they are informed about the relevance of a particular piece of information to the solving of the target problem. Therefore, it is not only considered theoretically but also pragmatically important to determine to what extent people in various cultures do in fact engage in analogical mapping when they are presented with a target and source analogue.

The present study aimed to examine transfer mechanisms and identify the factors that optimize transfer ability. Based on Chen's (2002) study it investigated how the process of analogizing takes place when the source is pictorially depicted and shares different levels of similarity (Principle, Strategy \& Procedural) with the target.

\section{Statement of the Problem}

Is transfer performance a function of the degree to which a source model and a target problem shared a similar solution procedure?

\section{Hypothesis}

The procedural level of similarity will influence the transfer performance of the participants more than the principle and strategy levels in solving the target problem.

The questions of the present study are:

- What is the effect of procedural similarity pictorially depicted on transfer?

- Are there any specific difficulties related to the pictorial representation that affect analogical transfer performance?

Although no cross cultural study was conducted, it was speculated that there is a possibility that the results might be different to e.g. North American under graduates in the nature and the extent to which analogical reasoning is used as an effective cognitive tool in a sample of undergraduate female students from in a Saudi Arabian university.

The level of procedural similarity between problems is conceptualized in a hierarchical representation Chen (2002).
The major prediction was that transfer performance would prove to be a function of the degree to which a source model and a target problem shared a similar solution procedure. Information in terms of only a general principle of the source problem was expected to be inadequate for analogical transfer because participants would have little information about how the principle could be implemented in the target problem. The higher the degree of concreteness shared by a source and the target solution in terms of a similar procedure, the higher the level of subsequent transfers that would result. This is presumed obviously because high superficial and structural similarity between the two problems facilitate more directly and readily the transfer process

The levels of the similarity or abstraction as described by Chen (2002) are:

The principle level of similarity (General idea) depicted only the superordinate concept in the source model, that is, the general relation between a large object and a set of smaller objects. No concrete information concerning how to achieve this comparison was given.

The similar principle but dissimilar strategy models (Seesaw balance) contained a specific strategy and procedure that illustrated the weight equivalence principle However, the model (seesaw balance) idea were not similar to that one required for the target problem at either the strategy or procedure level.

The strategy level of similarity (spring compression) illustrated concrete procedures similar to those required for the target problem at a strategy level but not at a procedure level.

The procedural level of similarity (sinking compression) depicted specific procedures that can be used to solve the target problem (Appendix A).

\section{Participants}

One hundred and fifty six undergraduate Saudi Arabian students enrolled in psychology and sociology classes at King Abdul Aziz University served as participants in this experiment and gained course credit for it.

\section{Materials}

A problem solving booklet, containing the source pictorial schematic models (Appendix A) and the target problem which was adapted from a traditional Chinese tale, "Weigh the Elephant" problem (Appendix B), was translated in to the Arabic language. It describes a scenario in which a boy needs to weigh an elephant but cannot find a scale big enough to weigh it (Chen, 2002). The subjects were asked to generate possible solutions for obtaining the weight of the elephant. The critical item was a boat. This was presented along with other relevant items such as a small scale, rocks, and various other small objects that were introduced naturally in the story for generating the solution. Pictures of these relevant objects, along with some other irrelevant items (e.g., table, containers, and boxes), were provided in the problemsolving booklet (Appendix C). These elements were added to differentiate between the participants who choose the key elements from those who did not. 
In this study, only one possible and appropriate solution of the target problem: the boat solution (sinking compression solution) was considered.

The steps of the boat solution are:-

- Put the elephant on a boat.

- Mark the water level on the boat.

- Replace the elephant with some smaller objects (e.g., rocks or containers) so that the water level reaches the mark.

- Weigh the smaller objects separately with the small scale.

- Sum the weight of small objects to get the weight of the elephant.

Five experimental and one control group to investigate more specifically only the effect of the three levels of similarity on transfer effectiveness were used.

Subjects were randomly assigned to six groups to compare a between subjects effects of levels of abstraction. The control group were given a green square. The experimental condition consisted of five groups on the basis of whether the source model contained a concrete procedure, where Group 1 has no concrete procedure Groups 2 to 5 were given a concrete procedure that differed in the degree to which the source model was similar to the target solution. Here Groups $2 \& 3$ were given a general idea in terms of similar principle and a dissimilar strategy respectively of the solution required for solving the target. Group 4 was given a strategy but dissimilar procedure and finally group 5 was given the specific procedure similar to that required by the target. Thus the five experimental conditions were determined by the relations (similarity level) shared between a given source model and the solution required by the target problem.

\section{Procedure}

The experiment was conducted in the following way:

A booklet depicted the source pictorial schematic model and the target problem. Participants were seated far apart and tested as individuals in groups of 12 to 15 .

Participants viewed a schematic picture serving as the source model and were instructed to interpret and write down its possible meanings within 3 minutes. In the second problem, participants were instructed to turn to the page containing the target story problem and were asked to read the story problem carefully within 80 seconds and to generate any solution they thought appropriate using only the objects illustrated on the next page within 200 seconds.

All participants were required to give a written answer to a few questions at the end of the booklet to evaluate whether the source picture was helpful and useful in solving the target problem, and to mention the type of difficulties they faced while solving the problem.

\section{Scoring}

The scoring of the responses were two folds:

Source model: A correct and complete answer for the interpretation of the source model received a score of (1) while an incorrect or incomplete answer received a score of (0). An appropriate and complete solution is one which included the idea that smaller objects can equal the weight of a larger item.

Target problem: a four-point efficiency scale 0-3 was developed by the researcher to analyze more deeply the different implementation methods revealed in target problem solving performance. The answers of each participant were scored as follows:

A person would score three points if the answer was complete and successful in solving the target problem. For example, in the case of the boat solution, a complete answer would involve putting the elephant on the boat and marking the water level on the boat, and replacing the elephant with rocks or other smaller items such as containers or boxes until the water surface reached the mark, and weighing the smaller items separately with the small scale. A score of two points for a solution strategy but not complete procedural solution to the target problem. An example is put the elephant on one side of the boat and the small rocks on the other. One point was given to a participant if she had a general idea (principle) of the meaning of weight without a strategy and procedure for solving the target problem. Here, for example, an answer would be considered to be a general solution if it contained the idea of estimating the elephant's weight by comparing it with the weights of other smaller objects but did not include an explanation of how to implement this principle. Finally a score of 0 was given to an incorrect answer.

\section{Reliability}

Two condition-blind observers independently scored the problem-solving performance of 37 subjects. The Pearson's correlation between them was $r=.89$, which was significant at the 0.01 level, thereby indicating a high inter-scorer reliability.

\section{Statistical Analysis}

The experiment involved five experimental conditions of levels of similarity and one control group as independent variables. The dependent variable was measured in two ways: the number of participants solving the source problem (score 1) and their performance on the target problem in terms of an efficiency score on a four point scale which ranged from 0 to 3 . Here a score of zero clearly indicates a no or completely irrelevant answer. Percentages were used to compare solvers and non-solvers in all conditions. One-way ANOVA for the participant scores on the efficiency scale of 0-3 was used to determine if there was a significant main effect of levels of similarity on target performance.

\section{Results}

Data analysis focused on the major concern of this study, that is whether the participants understand the source problem and were able to transfer this knowledge to the target problem. The analysis also tried to find out which level of similarity (principle, strategy or procedural) helped most the mapping process from the source to the target problem. Accordingly, the primary dependent measures of transfer were whether the source problem was interpreted correctly and applied to the target problem. 
Table 1 shows the results of complete solvers of source and target problems based on the first measure. The principle group $(11.5 \%)$ did not differ much from the similar strategy $(12 \%)$ and procedural $(10.7 \%)$. The control group, on the other hand, came up with $7.4 \%$ solvers. These differences among the number of source and target solvers in the six groups was found not significant $\chi 2(5, \mathrm{~N}=14)=1.43, \mathrm{p}=0.92$.

The one way ANOVA results also indicated no significant main effects of levels of similarity on target performance $\mathrm{F}(5,155)=0.340 \mathrm{p}=0.888$ thereby rejecting the hypothesis that procedural level of similarity will influence performance on the target problem more than other levels.

One of the aims of the experiment was also to find out the type of difficulties (constraints) that were experienced by the participants during problem solving. A qualitative analysis, of the written answers to some retrospective questions asked after the completion of the test, was undertaken. The most common difficulties reported were ambiguity $43.1 \%$ (need more clarity in pictures), $31.4 \%$ needed more time and $25.5 \%$ needed other or more tools to solve the target problem (Table2).

TABLE 1 The number of complete solver for both the source and target problems.

\begin{tabular}{|c|c|c|c|}
\hline $\begin{array}{c}\text { The Level of } \\
\text { Similarity }\end{array}$ & The Conditions & Target solver & Target non-solvers \\
\hline \multirow{2}{*}{ principle } & source solver & $3(11.5 \%)$ & $15(57.7 \%)$ \\
\cline { 2 - 4 } & source non-solver & $0(0 \%)$ & $8(30.7 \%)$ \\
\hline \multirow{2}{*}{ Seesaw bal. } & source solver & $1(4 \%)$ & $22(88 \%)$ \\
\cline { 2 - 4 } & source non-solver & $0(0 \%)$ & $2(8 \%)$ \\
\hline \multirow{2}{*}{ Hanging bal. } & source solver & $2(8 \%)$ & $13(52 \%)$ \\
\cline { 2 - 4 } & source non-solver & $1(4 \%)$ & $9(36 \%)$ \\
\hline \multirow{2}{*}{ Spring comp. } & source solver & $3(12 \%)$ & $12(48 \%)$ \\
\cline { 2 - 4 } & source non-solver & $1(4 \%)$ & $9(36 \%)$ \\
\hline \multirow{2}{*}{ Sinking comp. } & source solver & $3(10.7 \%)$ & $4(14.3 \%)$ \\
\cline { 2 - 4 } & source non-solver & $2(7.1 \%)$ & $19(67.8 \%)$ \\
\hline \multirow{2}{*}{ Control } & source solver & $2(7.4 \%)$ & $22(81.4 \%)$ \\
\cline { 2 - 4 } & source non-solver & $0(0 \%)$ & $3(11.1 \%)$ \\
\hline
\end{tabular}

TABLE 2 Type of difficulties

\begin{tabular}{|l|l|l|l|}
\hline Conditions & Ambiguity & Tools & Need more time \\
\hline complete solution & $18(14 \%)$ & $7(5.1 \%)$ & $3(2.9 \%)$ \\
\hline seesaw balance & $23(17.8 \%)$ & $5(3.6 \%)$ & $7(1 \%)$ \\
\hline hanging balance & $15(11.6 \%)$ & $16(11.7 \%)$ & $2(2 \%)$ \\
\hline spring compression & $22(17 \%)$ & $7(5.1 \%)$ & $6(5.98 \%)$ \\
\hline Total & $78(43.1 \%)$ & $35(25.5 \%)$ & $43(31.4 \%)$ \\
\hline
\end{tabular}

\section{Discussion}

Chen (2002) addressed some important theoretical issues regarding the effectiveness of the process of executing a process solution (implementing a solution in the target derived from the source problem) as greatly determined by the overall relations between the source analogue and its target problem. He held that when a learned source solution is similar to the target problem at only a conceptual level (giving only a principle or a general idea) the probability of transfer is very low. An intermediate level (strategy) of similarity between the source and target (where a solution process is given which differs from that required to solve the target) was also found to create difficulties though it was more effective than the conceptual level. But when the source and the target shared procedural details the transfer performance was increased.

The findings of this experiment were not entirely in the expected direction thereby rejecting the hypothesis that procedural similarity will have a significant positive effect on transfer performance as compared to other types of similarity.

In this experiment The source problem was encoded correctly by more than half of the participants $(60.5 \%)$. However, only $9 \%$ of them were able to solve the target problem successfully by applying the procedure from the source problem. This indicates that most of the participants had understood the source problem in itself but failed to map it to the target problem. Another important finding of the experiment is that $(92 \%)$ of the participants within the similar principle group (seesaw balance) interpreted the source problem correctly. This high percentage is perhaps due to the fact that this type of balance is commonly used for weighing in Saudi culture. On the other hand, contrary to the expectation only $25 \%$ of the participants within the similar procedure group (sinking compression) interpreted the source problem as a method of weighing. This could be attributed to the possibility that participants failed to understand the sinking compression type of balance due to unfamiliarity.

Mapping is considered an important stage where knowledge about the source is carried over to the target. The second level of analysis related to the solution of the target problem which required firstly identifying the goal (weighing the elephant), and secondly, transferring the relevant information from the source problem after encoding it and mapping it to the target problem. There was not much variation in the performance of the participants in the five experimental conditions and the control group in solving the target problem. It was found that the number of participants in the similar principle group (seesaw balance) solved the target problem procedurally correct which is almost equivalent to the number of participants in both the similar strategy (spring compression) and the similar procedure (sinking compression) groups. This finding is contrary to the expectation because the spring and sinking compression groups, of similar strategy and similar procedure respectively, were expected to perform better than the other groups. This lack of difference in the performance of the groups could be due to the fact that the participants in the similar strategy and similar procedure did not transfer the procedural knowledge from the source to the target perhaps because they failed to understand the concept of weighing by compression.

The findings of this experiment were not entirely in the expected direction also perhaps because the pictures presented 
in the source problem were misinterpreted by $40 \%$ of the participants reportedly either due to ambiguity (example the objects in the pictures were interpreted by some as people a part of a family or group waiting for the elevator) or perceiving the direction of pictures from $\mathrm{R}$ to $\mathrm{L}$ (cultural mental set) instead of $\mathrm{L}$ to $\mathrm{R}$.

\section{Conclusion}

This experiment based on Chen's study (2002) focused on the extent to which the solution of the target problem is a function of the five experimental conditions representing different levels of similarity hierarchically.

The results do not indicate a definite trend in the expected direction. They apparently did not support the prediction that the ease with which a source solution is implemented is largely determined by the abstraction level (representation) at which a solution is shared by a analogue and a target problem. The findings of the experiment also indicated the importance of culturally relevant information particularly in pictorial representations.

Thus the rather confounding and inconclusive results were attributed to some problems related to the understanding of the concepts and the process that was pictorially represented rendered this experiment a more exploratory purpose which makes it imperative that another experiment be conducted, taking into account both the direction and the clarity of the pictures depicting a process.

A follow up experiment was conducted using think aloud protocols to determine the extent to which the both the tendency of Arab students to process or perceive information in the $\mathrm{R}$ to $\mathrm{L}$ direction and the enhanced clarity of pictures in the source problem at all the three levels of similarity (principle, strategy and procedure) affected the process of transfer in solving the target problem.

\section{Acknowledgment}

I am grateful to Dr. Tahra Alaa-Dean for all the help, support and reviewer of an earlier version of this manuscript.

\section{References}

[1] S, Ainsworth. and A. Th Loizou, The effects of self-explaining when learning with text or diagrams. Cognitive Science, 2003. 27(4): p. 669-681.

[2] Chen, Z., Analogical Problem Solving: A Hierarchical Analysis of Procedural Similarity. Journal of Experimental Psychology: Learning, Memory, and Cognition, 2002. 28(1): p. 81-98.

[3] Chen, Z. and L. Mo, Schema Induction in Problem Solving: A Multidimensional Analysis,. Journal of Experimental Psychology: Learning, Memory, and Cognition, 2004. 30(3): p. 583-600.

[4] Daives, J., A.K. \& Goel, et al. (2003).Visual rerepresentation in creative analogies. International Joint Conference on Artificial Intelligence. Acapulco, Mexico.

[5] Gentner, D. "Structure-mapping: A theoretical framework for analogy". Cognitive Science, 1983. 7(2): p. 155-170.

[6] Gentner, D. \& Markman, A. (1993). Structure alignment during similarity comparison. Cognitive Psychology, 25, 431-467.

[7] Gentner, D., \& Markman, A.B. (1997). Structure mapping in analogy and similarity. American Psychologist, 52(1), 45-56.
[8] Gick, M.L., \& Holyoak, K.J. (1980). Analogical problemsolving. Cognitive Psychology, 12(3), 306-355.

[9] Gick, M.L., \& Holyoak, K.J. (1983). Schema induction and analogical transfer.Cognitive Psychology, 15(1), 1-38.

[10] Holyoak, K.J. \& Thagard, P. (1995).Mental leaps: Analogy in creative thought. MIT Press

[11] Kurtz, K. J., Boukrina, O., \& Gentner, D. (2013). Comparison promotes learning and transfer of relational categories. Journal of Experimental Psychology: Learning, Memory, and Cognition, 39(4), 1303.

[12] Markman, A.B. \& D. Gentner (2000). Structure mapping in the comparison process. American Journal of Psychology, 113, 501538.

[13] Van Meter, P., et al., Learner-generated drawing as a strategy for learning from content area text. Contemporary Educational Psychology, 2006. 31(2): p. 142-166.

[14] Zhang, J. and D.A. Norman, Representations in distributed cognitive tasks. Cognitive Science, 1994. 18(1): p. 87-122.

\section{Appendix A}
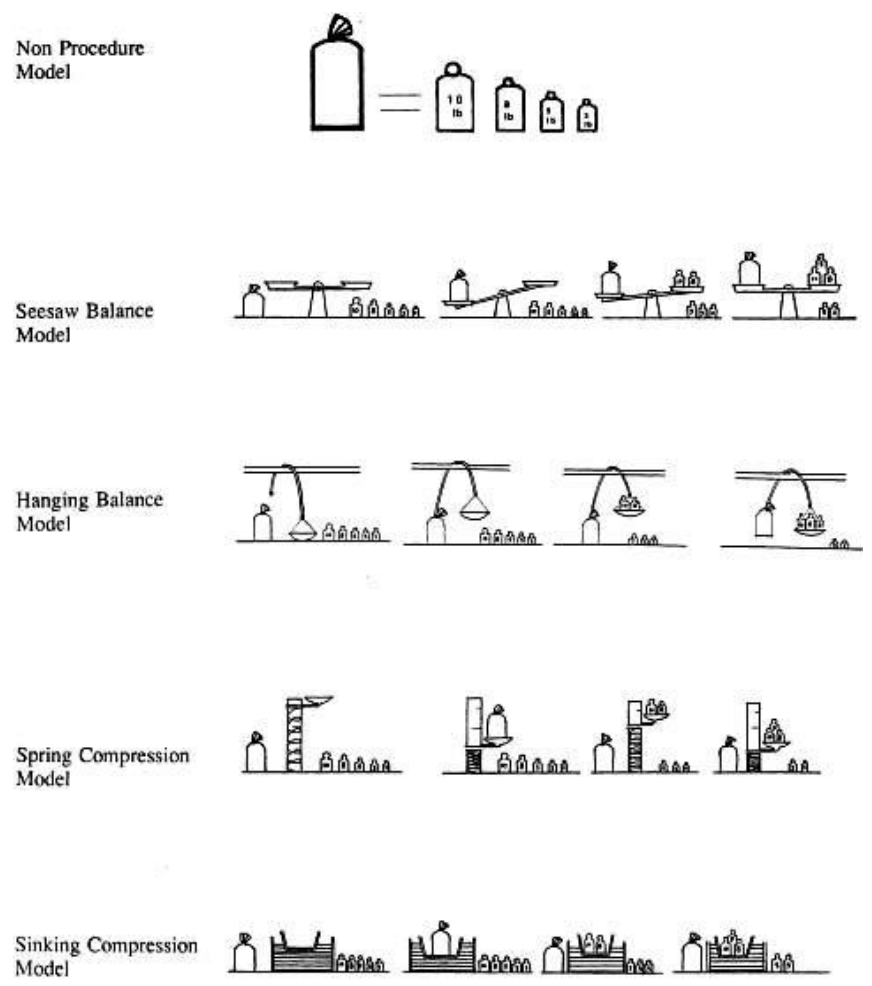

Figure 3.1illustrates the representation of the various schematic models in the source

- The general principle illustrates only the notion of weight equivalence without a concrete solution strategy or procedure for the target.

- The seesaw balance (similar principle) also illustrates the principle of weight equivalence in relatively more detail but without a concrete solution strategy or procedure for the target

- The hanging balance model gives a solution strategy but which is dissimilar to the target solution.

- The spring compression models depict a similar strategy to the target solution that several smaller objects can push down a compressible surface to the same degree as one heavy object. 
- The sinking compression model provides a similar procedure for the solution of the target.

\section{Appendix B}

\section{"Weigh the Elephant" Problem}

Many years ago there lived in China a young man. Wishing to further his education, he went to a wise man in a remote land.

"Master," he said, "if you will allow me to study with you for one year, I will give you, in payment, this elephant." And he displayed to the wise man an elephant, strong and beautiful.

The old man looked from the young man to the elephant, and asked: "How much does the elephant weigh?"

"I do not know, Master" the boy replied.

"Weigh the elephant. Come back tomorrow and we will begin to learn from each other."

So the boy left, running through the town, looking for a scale to weigh the elephant. The largest scale he could find, however, was only scaled to 200 pounds.

"The next morning the boy sat, despondent, under a big tree, on a rocky river bank. As he watched, a boat came into view; the old man was rowing toward him. The old man got out of the boat, went to the boy and sat down.

"How much does your elephant weigh?"

"I cannot find a large scale, master."

"It is not the elephant I am measuring, my son. It is the student's thinking. You have everything you need to weigh the elephant. When you have done so, you may join me." And the old man stood up and moved up the path to his school, leaving the boy with the problem (Chen, 2002).

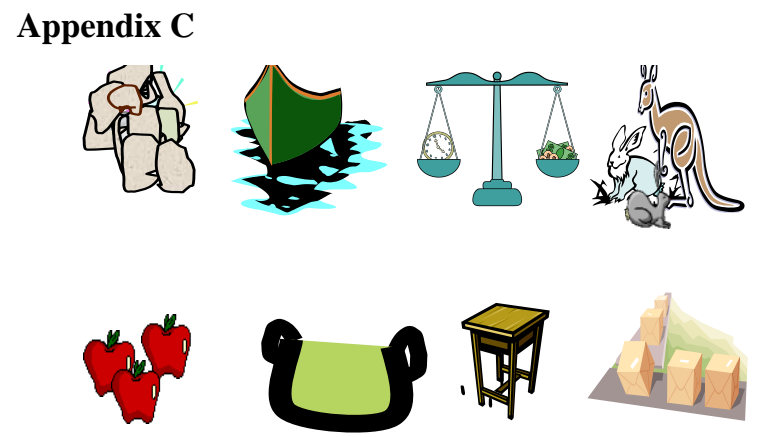

